\title{
Association of inflammatory gene polymorphisms with ischemic stroke in a Chinese Han population
}

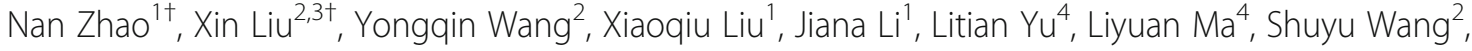 \\ Hongye Zhang ${ }^{2}$, Lisheng Liu'2, Jingbo Zhao ${ }^{1 *}$ and Xingyu Wang ${ }^{2,3^{*}}$
}

\begin{abstract}
Background: Inflammatory mechanisms are important in stroke risk, and genetic variations in components of the inflammatory response have been implicated as risk factors for stroke. We tested the inflammatory gene polymorphisms and their association with ischemic stroke in a Chinese Han population.

Methods: A total of 1,124 ischemic stroke cases and 1,163 controls were genotyped with inflammatory panel strips containing 51 selected inflammatory gene polymorphisms from 35 candidate genes. We tested the genotype-stroke association with logistic regression model.

Results: We found two single nucleotide polymorphisms (SNPS) in CCL11 were associated with ischemic stroke. After adjusting for multiple testing using false discovery rate (FDR) with a 0.20 cut-off point, CCL11 rs4795895 remained statistically significant. We further stratified the study population by their hypertension status. In the hypertensive group, CCR2 rs1799864, CCR5 rs1799987 and CCL11 rs4795895 were nominally associated with increased risk of stroke. In the non-hypertensive group, CCL11 rs3744508, LTC4S rs730012, FCER1B rs569108, TGFB1 rs1800469, LTA rs909253 and CCL11 rs4795895 were associated with ischemic stroke. After correction for multiple testing, CCR2 rs1799864 and CCR5 rs1799987 remained significant in the hypertensive group, and CCL11 rs3744508, LTC4S rs730012, FCER1B rs569108, TGFB1 rs1800469, LTA rs909253 remained significant in the non-hypertensive group.

Conclusions: Our results indicate that inflammatory genetic variants are associated with increased risk of ischemic stroke in a Chinese Han population, particularly in non-hypertensive individuals.
\end{abstract}

Keywords: Association study, Hypertension, Inflammatory gene, Ischemic stroke

\section{Background}

Stroke is one of the most common causes of mortality and a leading cause of adult disability worldwide [1]. In China, stroke is the leading cause of death, and ischemic stroke accounts for two-thirds of all strokes [2]. To date, although the etiology and mechanisms of stroke have not been well understood, it is considered as a complex multifactorial disorder with an interaction between the individual's genetic background and various environmental factors. Previous studies have demonstrated that high blood pressure, smoking, poor diet, abdominal

\footnotetext{
* Correspondence: zhaojb168@sina.com; xingyuw@yahoo.com ${ }^{\dagger}$ Equal contributors

'Department of Epidemiology, Public Health School, Harbin Medical University, Heilongjiang, China

${ }^{2}$ Laboratory of Human Genetics, Beijing Hypertension League Institute, Beijing, China

Full list of author information is available at the end of the article
}

obesity, and lack of physical activities as five common risk factors associated with $80 \%$ of all strokes [3]. However, these conventional stroke risk factors do not fully account for the overall risk of stroke. Growing evidence suggests inflammatory processes play fundamental roles in both the etiology and pathophysiology of ischemic cerebrovascular disease [4]. Inflammatory molecules, as well as single nucleotide polymorphisms (SNPs) of genes encoding inflammatory mediators, contribute to the development and progression of a large number of pathological conditions, including cardiovascular diseases [5]. SNPs of inflammatory genes strongly influence the plasma levels and biological activity of the corresponding proteins, with potentially important clinical implications $[6,7]$. In this study, we investigated the association between history of ischemic stroke and polymorphisms of genes encoding prototypical inflammatory molecules, 
such as interleukin $4(I L-4)$, interleukin 6 (IL-6), intercellular adhesion molecule 1 (ICAM-1), E-selectin (E-sel), chemokine (C-C motif) ligand 11 (CCL11), lymphotoxin $\alpha(L T A)$, and so on.

Although hypertension is the most significant risk factor for stroke [3], evidence from animal and human studies has indicated that some genes predisposing to ischemic stroke are independent of blood pressure [8]. In order to better control the confounding effect of blood pressure in ischemic stroke, the case and control subjects were stratified by their hypertension status.

\section{Materials and methods Study participants}

The Stroke Hypertension INvestigation IN Genetics (SHINING) study was conducted by Beijing Hypertension League Institute from 1997 to 2000. SHINING study comprised of subjects exclusive to Han ethnicity. A total of 3,119 participants (1,559 stroke cases and 1,560 controls) were recruited from 6 geographical regions in northern China. Cases were recruited from the community of those who were discharged from hospitals between 1997 to 2000; any stroke patients who suffered a stroke within the past 5 years were eligible to participate the study. All patients had to have medical records with diagnosis from brain computed tomography (CT)/MRI. Control subjects were selected according to the case-control study criteria during the same period (control subjects matched to cases by sex, age within 3 years, geographic location, and blood pressure category $(<140 / 90, \quad \geq 140 / 90$ and $\leq 180 / 105, \quad>180 /$ $105 \mathrm{mmHg})$ ). Details of the protocol have been described elsewhere [9]. Cases with a prior history of myocardial infarction as well as controls with a previous history of myocardial infarction or stroke were excluded.

In this study, we focused only on ischemic stroke $(1,124$ ischemic stroke cases and 1,163 controls) since it is the most prevalent form of stroke that accounts for more than three-quarters of all cases. Data collected included age, sex, body mass index (BMI), systolic blood pressure (SBP), diastolic blood pressure (DBP) and hypertension. Blood pressures were either measured (one to three measurements) or extracted from medical records. Hypertension was defined as systolic blood pressure $\geq 140 \mathrm{mmHg}$ or diastolic blood pressure $\geq 90 \mathrm{mmHg}$ or using of antihypertensive medication.

All participants had signed written informed consent and the study was approved by the ethics committee of Beijing Hypertension League Institute.

\section{Selection of SNPs and genotyping}

DNA was extracted from the whole blood with salting out procedure. We used a polymerase chain reaction (PCR)based inflammatory marker panel (Roche Molecular
Biochemicals, Basel, Switzerland) to genotype DNA samples. Basic information on the selected SNPs is shown in Additional file 1: Table S1. These SNPs are not in linkage disequilibrium. The linkage disequilibrium calculation was performed using Haploview 4.1 software. The SNPs on the strip system represent a selection of genetic variants, essentially all of which had been associated with inflammatory diseases before the development of the strips. The detailed genotyping procedure was described previously [9]. In brief, multilocus PCR was carried out in a single tube containing 51 pairs of biotinylated primers. Each amplified PCR product was hybridized with sequencespecific oligoprobes immobilized on a nylon membrane strip; biotin-based color was developed and captured with a scanner and proprietary software developed by Roche Molecular Systems. Genotyping calls were made by two researchers to yield unanimous results. The call rates of SNPs were more than $98 \%$.

\section{Statistical analysis}

Continuous variables were expressed as mean \pm SD and differences between case and control groups were analyzed by Student's $t$ test. Categorical variables were presented as percentage. The $\chi^{2}$ or exact test was used to identify significant departure from Hardy-Weinberg equilibrium. Polymorphisms with a minor allele frequency (MAF) $<5 \%$ would be excluded from the analyses.

Logistic regression models were used to estimate odds ratios (ORs) and their 95 percent confidence intervals (95\% CIs) by using an additive genetic model. An additive model was built that assumed that the risk for carriers of the heterozygous genotype for developing the outcome was half way between carriers of the homozygous genotypes. The advantage was that the strength of genotype-phenotype association was expressed in a single parameter (best estimate) and statistical tests had only 1 degree of freedom [10]. Also this model would have sufficient power to capture dominant modes of transmission given our sample size. In a first step, ageadjusted and sex-adjusted models were constructed. Thereafter, we fitted multivariable models adjusting for age, sex, BMI and hypertension status. Given the similarity of the results, only multivariable adjusted models are presented in this manuscript. To adjust for multiple hypothesis testing, the false discovery rate (FDR) was applied [11]. The FDR significance threshold was defined with a value of 0.20 [12], meaning that one should expect at most $20 \%$ of declared discoveries to be false. A multivariate analysis was performed to find interactions between polymorphisms and hypertension status, adjusting for age, sex, BMI. All analyses were performed using SAS V.9.1.3 (SAS, Cary, NC, USA).

Power calculations were generated using a log-additive model of risk, $5 \%$ type 1 error rate. For a minor allele 
frequency of 0.05 , the power in the discovery phase of total group was $86.46 \%$; the power in the hypertension group was $65.50 \%$; and in non-hypertension the power was $57.34 \%$. Power calculations were performed using Quanto software (http://hydra.usc.edu/gxe/).

\section{Results}

The baseline and clinical characteristics of study participants are presented in Table 1 . There were 1,124 ischemic stroke cases and 1,163 controls in this study. The control group was older than the case group. There were more individuals with hypertension in the control group and BMI was slightly higher compared to the case group.

Among the 51 polymorphisms, 11 SNPs had a MAF $<5 \%$. Two SNPs (interleukin 4 (IL4) rs2243250 $(P=0.001)$ and nitric oxide synthase 3 (endothelial cell) (NOS3) rs1800779 $(P=0.012)$ ) deviated from HardyWeinberg equilibrium (HWE) in the control group and were excluded from subsequent analysis. Thus, 38 SNPs were selected for further analysis (see Additional file 1: Table S1).

As shown in Table 2, two SNPs in CCL11 were associated with ischemic stroke $(P<0.05)$ in total study population. The ORs (95\% CI; $P$ value) were 1.47 (1.13 1.92; 0.004) for CCL11 rs4795895 and 1.20 (1.01-1.43; 0.042 ) for CCL11 rs3744508. After adjustment for multiple testing, CCL11 rs4795895 remained significant $(\mathrm{FDR}=0.152)$.

Hypertension is a major risk factor for stroke. Evidence has shown that stroke patients with or without hypertension exhibited different clinical features and different pathophysiology [13]. In consideration of the potential effect, hypertension stratification was decided on a priori. Odds ratios for the incidence of ischemic stroke are shown in Table 3. After stratification by hypertension and adjustment for age, sex, and BMI, 3 of 38 SNPs showed significant association $(\mathrm{P}<0.05)$ with ischemic stroke in the hypertensive group. These SNPs were in chemokine (C-C motif) receptor 2 (CCR2), chemokine

Table 1 Characteristics of study participants

\begin{tabular}{|c|c|c|c|}
\hline & Ischemic stroke patients & Controls & $P$ value \\
\hline Number of subjects & 1,124 & 1,163 & \\
\hline Age, mean (SD), years & $59.20(10.71)$ & $62.32(10.68)$ & $<0.0001$ \\
\hline Male, \% & 59.82 & 61.25 & 0.483 \\
\hline BMI, mean (SD), $\mathrm{kg} / \mathrm{m}^{2}$ & $24.42(3.00)$ & $25.04(3.28)$ & $<0.0001$ \\
\hline Hypertension, \% & 64.74 & 70.19 & 0.005 \\
\hline \multicolumn{4}{|c|}{$\begin{array}{l}\text { Data presented as percentage of participants unless otherwise stated. } P \text { values } \\
\text { for } \chi^{2} \text { test for categorical variables, and Student's } t \text { test for continuous } \\
\text { variables. Hypertension: systolic blood pressure } \geq 140 \mathrm{mmHg} \text { and/or diastolic } \\
\text { blood pressure } \geq 90 \mathrm{mmHg} \text {, or current treatment with antihypertensive } \\
\text { medication. } \\
\text { BMI = body mass index. }\end{array}$} \\
\hline
\end{tabular}

(C-C motif) receptor 5 (CCR5) and CCL11. The ORs (95\% CI; $P$ value) were 1.26 (1.06 - 1.49; 0.009) for CCR2 rs1799864, 1.23 (1.06 - 1.42; 0.007) for CCR5 rs1799987 and $1.45(1.05-1.99 ; 0.023)$ for CCL11 rs4795895. The FDR values were $0.152,0.152$ and 0.291 , respectively.

In the non-hypertensive group, after adjustment for age, sex and BMI, there were six SNPs associated with stroke. The ORs (95\% CI; $P$ value) of these SNPs were 1.64 (1.20 - 2.24; 0.002) for CCL11 rs3744508, 1.56 (1.14 - 2.14; 0.006) for leukotriene C4 synthase (LTC4S) rs730012, 1.48 (1.02 - 2.00; 0.009) for FcepsilonRI- $\beta$ (FCER1B) rs569108, 1.28 (1.03 - 1.59; 0.028) for lymphotoxin $\alpha$ (LTA) rs909253, $1.30(1.07$ - 1.59; 0.009) for transforming growth factor $\beta 1$ (TGFB1) rs1800469 and 1.67 (1.05 - 2.66; 0.032) for CCL11 rs4795895. After correction for multiple testing, five SNPs, CCL11 rs3744508, LTC4S rs730012, FCER1B rs569108, TGFB1 rs1800469, LTA rs909253, had FDR values less than 0.20 (Table 3). We tested the interaction between hypertension and genetic variants. There was no interaction detected between any genetic variants and hypertension status (data not shown).

\section{Discussion}

In this case-control study, we assessed the relationship of 51 SNPs in 35 genes related to inflammatory response with the risk of ischemic stroke in 1,124 cases and 1,163 controls. In the control group, there were more individuals with hypertension and BMI was slightly higher compared to the case group. This was due to our effort to recruit controls to match the cases with blood pressure in the search for genes predisposing to ischemic stroke with and without the influence of hypertension.

Initial analysis showed that CCL11 rs4795895 and CCL11 rs3744508 were significantly associated with ischemic stroke by adjusting for age, sex, BMI and hypertension status, and after adjusting for multiple testing by FDR, CCL11 rs4795895 remained significantly associated with ischemic stroke. The CCL11 rs4795895 genetic polymorphism was the only SNP that retained significant association with ischemic stroke in the whole group. After stratification by hypertension, SNP rs4795895 was still significant in both the non-hypertensive and hypertensive groups, though after adjusting for the multiple hypothesis test it was not significantly associated with ischemic stroke. Polymorphisms rs4795895 and rs3744508 were located in the $5^{\prime}$ flanking region and the coding region of the eotaxin 1 gene, respectively [14]. Previous reports have noted that the presence of the CCL11 rs3744508 A allele is associated with lower CCL11 production [15] and an increased risk of myocardial infarction [16]. Ours is the first study to report any association with SNPs of CCL11 and ischemic stroke. There are two possible mechanisms by which eotaxin 
Table 2 Association between single nucleotide polymorphisms (SNPs) and ischemic stroke

\begin{tabular}{|c|c|c|c|c|c|c|c|c|}
\hline \multirow[t]{2}{*}{ Gene } & \multirow[t]{2}{*}{ rsID } & \multirow{2}{*}{$\begin{array}{l}\text { Minor } \\
\text { allele }\end{array}$} & \multirow{2}{*}{$\begin{array}{l}\text { Risk } \\
\text { allele }\end{array}$} & \multirow{2}{*}{$\begin{array}{l}\text { MAF, } \\
\text { control: } \\
\text { case }\end{array}$} & \multicolumn{3}{|c|}{ Additive model } & \multirow[t]{2}{*}{ FDR } \\
\hline & & & & & $\mathrm{OR}$ & $95 \% \mathrm{Cl}$ & $P$ value & \\
\hline CCL11 & rs4795895 & A & G & 0.05:0.07 & 1.47 & $1.13-1.92$ & 0.004 & 0.152 \\
\hline CCL11 & rs3744508 & A & A & 0.14:0.12 & 1.2 & $1.01-1.43$ & 0.042 & 0.547 \\
\hline CCR5 & rs1799987 & A & A & $0.46: 0.43$ & 1.13 & $1.00-1.27$ & 0.051 & 0.547 \\
\hline TGFB1 & rs1800469 & C & C & $0.48: 0.49$ & 0.9 & $0.80-1.11$ & 0.071 & 0.547 \\
\hline NOS3 & rs1799983 & $\mathrm{T}$ & G & $0.10: 0.11$ & 1.19 & $0.99-1.45$ & 0.072 & 0.547 \\
\hline CCR2 & rs1799864 & A & A & $0.27: 0.25$ & 1.11 & $0.97-1.27$ & 0.143 & 0.614 \\
\hline IL6 & rs1800796 & G & G & $0.33: 0.31$ & 1.1 & $0.97-1.25$ & 0.144 & 0.614 \\
\hline ILAR & rs1801275 & G & G & $0.17: 0.16$ & 1.12 & $0.96-1.32$ & 0.162 & 0.614 \\
\hline$A D R B 2$ & rs1042713 & G & G & $0.44: 0.42$ & 1.09 & $0.96-1.23$ & 0.172 & 0.614 \\
\hline CD14 & rs2569190 & $\mathrm{T}$ & $\mathrm{T}$ & $0.37: 0.38$ & 1.09 & $0.96-1.23$ & 0.187 & 0.614 \\
\hline TNF & rs361525 & A & A & $0.05: 0.05$ & 1.2 & $0.90-1.61$ & 0.208 & 0.614 \\
\hline FCERB 1 & rs569108 & G & G & $0.17: 0.16$ & 1.11 & $0.94-1.30$ & 0.217 & 0.614 \\
\hline LTA & rs909253 & G & G & $0.42: 0.41$ & 1.08 & $0.95-1.22$ & 0.227 & 0.614 \\
\hline IL1A & rs1800587 & $\mathrm{T}$ & $C$ & 0.09:0.10 & 1.12 & $0.92-1.37$ & 0.258 & 0.614 \\
\hline LTC4S & rs730012 & C & C & $0.15: 0.14$ & 1.1 & $0.93-1.30$ & 0.263 & 0.614 \\
\hline ADRB2 & rs1042714 & G & G & 0.07:0.11 & 1.11 & $0.92-1.34$ & 0.267 & 0.614 \\
\hline$V D R$ & rs2228570 & $\mathrm{T}$ & $\mathrm{T}$ & $0.45: 0.43$ & 1.15 & $0.89-1.49$ & 0.282 & 0.614 \\
\hline IL10 & rs1800872 & $C$ & A & $0.35: 0.36$ & 1.07 & $0.95-1.21$ & 0.291 & 0.614 \\
\hline GC & rs7041 & G & G & $0.27: 0.26$ & 1.07 & $0.94-1.23$ & 0.321 & 0.642 \\
\hline$V D R$ & rs1544410 & A & A & 0.06:0.05 & 1.05 & $0.94-1.19$ & 0.387 & 0.735 \\
\hline IL5RA & rs2290608 & A & A & $0.24: 0.23$ & 1.06 & $0.92-1.22$ & 0.435 & 0.787 \\
\hline ILIB & rs16944 & $\mathrm{T}$ & $\mathrm{T}$ & 0.49:0.47 & 1.05 & $0.93-1.18$ & 0.456 & 0.788 \\
\hline TNF & rs1800629 & A & A & 0.06:0.06 & 1.09 & $0.86-1.39$ & 0.478 & 0.79 \\
\hline CTLA4 & rs5742909 & $\mathrm{T}$ & C & $0.13: 0.14$ & 1.06 & $0.89-1.26$ & 0.528 & 0.81 \\
\hline CSF2 & rs25882 & $\mathrm{T}$ & $C$ & 0.38:0.39 & 1.04 & $0.92-1.17$ & 0.533 & 0.81 \\
\hline TCF7 & rs244656 & $\mathrm{T}$ & A & $0.12: 0.13$ & 1.04 & $0.87-1.25$ & 0.649 & 0.856 \\
\hline ILAR & rs1805015 & $C$ & $C$ & 0.08:0.08 & 1.05 & $0.84-1.30$ & 0.669 & 0.856 \\
\hline SELP & rs6131 & A & A & $0.19: 0.20$ & 1.03 & $0.89-1.20$ & 0.674 & 0.856 \\
\hline IL13 & rs1295686 & $\mathrm{T}$ & C & $0.28: 0.29$ & 1.03 & $0.90-1.17$ & 0.679 & 0.856 \\
\hline VCAM1 & rs1041163 & C & $\mathrm{T}$ & 0.15:0.16 & 1.03 & $0.88-1.21$ & 0.687 & 0.856 \\
\hline CXCL12 & rs1801157 & A & G & $0.21: 0.22$ & 1.03 & $0.89-1.19$ & 0.698 & 0.856 \\
\hline CTLA4 & rs231775 & A & G & $0.31: 0.31$ & 1.02 & $0.90-1.16$ & 0.738 & 0.876 \\
\hline C5 & rs17611 & G & G & $0.42: 0.42$ & 1.02 & $0.90-1.15$ & 0.768 & 0.884 \\
\hline ILAR & rs1805010 & G & G & $0.48: 0.48$ & 1.01 & $0.90-1.14$ & 0.827 & 0.899 \\
\hline ICAMI & rs5491 & $\mathrm{T}$ & $\mathrm{T}$ & 0.07:0.06 & 1.03 & $0.80-1.31$ & 0.828 & 0.899 \\
\hline$U G B$ & rs3741240 & A & A & $0.43: 0.43$ & 1.03 & $0.89-1.13$ & 0.965 & 0.977 \\
\hline NOS2A & rs1137933 & $\mathrm{T}$ & $\mathrm{T}$ & $0.13: 0.13$ & 1.00 & $0.84-1.19$ & 0.97 & 0.977 \\
\hline GC & rs4588 & A & $A$ & 0.32:0.32 & 1.00 & $0.88-1.14$ & 0.977 & 0.977 \\
\hline
\end{tabular}

OR was calculated using a reference allele that resulted in an OR $>1 . P$ value and FDR adjusted for age, sex, body mass index and hypertension status in additive model of inheritance.

$\mathrm{FDR}=$ false discovery rate; $\mathrm{MAF}=$ minor allele frequency . 
Table 3 Single nucleotide polymorphisms (SNPs) achieving $\boldsymbol{P}<\mathbf{0 . 0 5}$ for association with ischemic stroke based on hypertension-stratified population

\begin{tabular}{|c|c|c|c|c|c|c|c|c|}
\hline Gene & rsID & Minor allele & Risk allele & MAF, control:case & OR & $95 \% \mathrm{Cl}$ & $P$ value & FDR \\
\hline \multicolumn{9}{|c|}{ Non-hypertension } \\
\hline CCL11 & rs4795895 & A & G & 0.07:0.05 & 1.57 & $1.05-2.66$ & 0.032 & 0.202 \\
\hline CCL11 & rs3744508 & A & A & $0.10: 0.16$ & 1.64 & $1.20-2.25$ & 0.002 & 0.076 \\
\hline LTA & rs909253 & G & G & $0.39: 0.45$ & 1.29 & $1.03-1.60$ & 0.024 & 0.182 \\
\hline LTC4S & rs730012 & C & C & $0.11: 0.16$ & 1.55 & $1.13-2.12$ & 0.007 & 0.086 \\
\hline TGFB1 & rs1800469 & C & C & $0.46: 0.46$ & 1.30 & $1.07-1.59$ & 0.009 & 0.086 \\
\hline FCERB 1 & rs569108 & G & G & $0.12: 0.17$ & 1.49 & $1.11-2.00$ & 0.009 & 0.086 \\
\hline \multicolumn{9}{|c|}{ Hypertension } \\
\hline CCL11 & rs4795895 & A & G & 0.07:0.05 & 1.45 & $1.05-1.99$ & 0.023 & 0.291 \\
\hline CCR2 & rs1799864 & A & A & $0.23: 0.28$ & 1.26 & $1.06-1.49$ & 0.008 & 0.152 \\
\hline CCR5 & rs1799987 & A & A & 0.43:0.48 & 1.23 & $1.06-1.42$ & 0.007 & 0.152 \\
\hline
\end{tabular}

Non-hypertension: cases $=397$ controls $=347$; hypertension: cases $=727$ controls $=816$. OR was calculated using a reference allele that resulted in an OR $>1$. $P$ value and FDR adjusted for age, sex and body mass index in additive model of inheritance.

$\mathrm{FDR}=$ false discovery rate; $\mathrm{MAF}=$ minor allele frequency.

might be related to stroke risk. First, it may be the direct consequence of atherosclerosis development. The eotaxin gene and protein are over expressed in human atherosclerosis [17]. Vascular smooth muscle cells (VSMCs) [18] and endothelium [19] in human atheroma prominently express eotaxin, suggesting that eotaxin contributes to the progression of atherosclerosis. Second, increased eotaxin levels can mirror the development of inflammatory responses in heart and brain. Farahi et al. reported that circulating eotaxin levels were increased in patients with coronary artery disease [15].

To explore the influence of inflammatory genes on ischemic stroke in the hypertension and non-hypertension groups, we stratified study subjects by their hypertension status and found that CCR2 rs1799864 and CCR5 rs1799987 polymorphisms were associated with ischemic stroke in the hypertensive group, and CCL11 rs3744508, LTC4S rs730012, FCER1B rs569108, TGFB1 rs1800469 and LTA rs909253 were associated with ischemic stroke in the non-hypertensive group.

CCR2 rs1799864, a SNP causing an isoleucine-forvaline substitution at position 64 in the CCR2 receptor on chromosome 3, was found to be associated with ischemic cardiomyopathy and myocardial infarction in Czech populations [20,21]. And in an African-American population from the Johns Hopkins Sibling and Family Heart Study, it was reported that the combination of homozygotic or heterozygote CCR2 rs1799864 genotype and monocyte chemoattractant protein 1 (MCP-1) rs2857656 homozygous genotype was associated with a particularly high prevalence of carotid artery plaque [22]. Chemokine receptor 2 (CCR2) is the receptor for $\mathrm{MCP}-1$. Recent studies have indicated that MCP-1 and $C C R 2$ could have an important role in regulating blood- brain barrier permeability. An animal study had indicated that a lack of CCR2 greatly reduced brain edema formation and $\mathrm{BBB}$ disruption, as well as decreasing leukocyte infiltration, and $C C R 2^{-1-}$ mice had decreased expression of a wide range of proinflammatory cytokines during reperfusion [23]. Polymorphism CCR5 rs1799987 is located at position 59,029 in the promoter region of CCR5 gene. The A allele of the SNP is associated with susceptibility to diabetic nephropathy [24]. Chemokine receptor 5 (CCR5) is the receptor for expressed on regulated on activation, normal $\mathrm{T}$ cell expressed and secreted (RANTES). RANTES was found as a mediator of cerebral ischemia-reperfusion (I/R) induced blood-brain barrier disruption, tissue injury, and the inflammatory and prothrombogenic phenotype assumed by the cerebral microvasculature after focal I/R [25]. In our study, CCR2 rs1799864 and CCR5 rs1799987 were associated with ischemic stroke only in the hypertensive group. We tested the interaction between the polymorphisms and hypertension status, and observed no relationship between them. A possible explanation is that in conditions of high blood pressure, patients carrying risk allele(s) suffer from cerebrovascular accident more than others due to direct or indirect effects of CCR2/CCR 5 on blood-brain barrier and blood vessel of brain.

CCL11, CCR2 and CCR5 belong to the CC family of chemokines. CC chemokines and their receptor polymorphisms have been shown to be associated with a number of infection or inflammation-related disease states, such as atherosclerosis [26]. To the best of our knowledge, our study is the first to demonstrate the association of CCL11, CCR2 and CCR5 genetic polymorphisms with the risk of ischemic stroke in a Chinese population. $\mathrm{CC}$ chemokines may play an important role 
in both the etiology and pathophysiology of ischemic stroke; the functional relevance of these SNPs to ischemic stroke remains to be further elucidated and CC chemokines could represent promising therapeutic targets in primary and secondary prevention of ischemic stroke.

In our study TGFB1 rs1800469, LTC4S rs730012 and LTA rs909253 were associated with ischemic stroke in the non-hypertensive group, which is in line with previous reports [9,27-30], although these findings drew from different ethnic populations and different stroke subtypes. Transforming growth factor $\beta$ (TGFB) is a pleiotropic cytokine with a diversity of effects, such as inducing or facilitating vascular stenosis and thrombogenesis [31,32]. TGFB1 rs1800469 polymorphism is located in the promoter region and considered possible modulator of expression of TGF- $\beta 1$ gene and levels. A case-control study had found an increased risk of stroke associated with the risk allele of the rs1800469 in a population from The Netherlands [27]. The rs730012 variant of leukotrieneC4 Synthase (LTC4S) upregulates LTC4S mRNA expression, increasing the synthesis of proinflammatory leukotrienes [28]. One study found that the LTC4S rs730012 variant contributed to large vessel stroke risk in children with sickle cell anemia [29]. Our previous study, a meta-analysis of association of candidate polymorphisms and ischemic stroke in six populations, indicated LTA rs909253 was associated with increased risk for ischemic stroke in normotensive subjects but not in hypertensive ones [9]. This is in concordance with the present report.

The $\beta$ subunit of high affinity IgE receptor (FCER1B) is a critical component of the IgE receptor, serving as a chaperone to promote assembly and expression and a strong amplifier of IgE-mediated signals [33]. The FCER $1 B$ rs569108 $\mathrm{G}$ allele was found to have an association with the development of allergic asthma in a Chinese population [30]. In our study, the $G$ allele of rs569108 increased the risk of ischemic stroke in nonhypertensive subjects. To explore the role of FCER1B polymorphism in pathogenesis of ischemic stroke, more genetic and functional studies will be needed.

There were five SNPs identified in the nonhypertension group and two SNPs in the hypertension group, however there were many more patients in the hypertension group. One possible explanation is that in the non-hypertensive group, the effect of genetic variation in inflammation gene may be easier to discern. Clinical and experimental studies have reported that hypertension itself is a chronic, low-grade inflammatory process [34]. High blood pressure levels are associated with an increase in circulating levels of inflammation markers that can reflect a vascular inflammatory process [35]. Hypertension could be a stronger factor causing inflammatory process. Therefore, it is possible that in the hypertensive group the genetic influence of inflammatory polymorphisms is concealed. Another possible explanation is that different ischemic stroke subtypes may occur in the different groups stratified by hypertensive status, and stratification could result in a more homogeneous population. According to Arboix et al.'s research [13], hypertension was the main cardiovascular risk factor only for lacunar and atherothrombotic infarction. In the present study, different significant SNPs were observed in the hypertensive and non-hypertensive groups, indicating that these polymorphisms may be associated with different subtypes of ischemic stroke, although we could not further define the subtypes. To the best of our knowledge, there is no study with the same design as ours, although hundreds of genetic studies of ischemic stroke have been performed in different geographic and ethnic populations. The ambiguous definition and stratification of ischemic stroke subtypes may be a major reason why no confirmed inflammatory genetic factor was found despite so many research projects on the genetics of ischemic stroke.

There are limitations to our study. First, the current study was a retrospective study, and the included stroke patients were stroke survivors. It could not exclude possible selection bias. Secondly, the cases were not further diagnosed with stroke subtypes, which may confound the results. Thirdly, due to the limitations of the study scope we did not collect information on subjects' diabetes status, hypercholesterolemia, and coronary artery disease, as well as plasma concentrations of inflammatory markers, which play an important role in the development of ischemic stroke. Also, smoking status was not included in the multivariable analyses. Finally, the results from this study have not been replicated, and should be further validated by a carefully designed study.

\section{Conclusions}

The current study made a survey of inflammatory gene polymorphisms in examining the risk for ischemic stroke. The results were largely concordant with previous knowledge of inflammation and vascular diseases. Our study demonstrated CCL11 rs4795895 is associated with ischemic stroke after adjusting for multiple testing. We also found five SNPs were associated with ischemic stroke in the non-hypertensive group and two SNPs were associated with ischemic stroke in the hypertensive group. These results collectively showed that inflammatory genetic variants were significantly associated with increased risk of ischemic stroke, particularly in nonhypertensive individuals. Additional studies in larger populations will be needed to confirm the relationship of inflammatory polymorphisms to stroke and its subtypes. 


\section{Additional file}

Additional file 1: Table S1. Risk allele frequencies, minor allele frequencies and Hardy-Weinberg equilibrium $P$ values for the genetic polymorphisms in the Stroke Hypertension INvestigation IN Genetics (SHINING) study.

\section{Competing interests}

The authors declare that they have no competing interests.

\section{Acknowledgements}

This study was supported by the Beijing Hypertension League Institute, in part through the National Infrastructure Program of Chinese Genetic Resource (2005DKA21300) and an unrestricted educational grant from F. Hoffmann-La Roche. We thank Ms Wei Zhang, Jian Li, and Jinli Xing for their excellent technical assistance.

\section{Author details}

'Department of Epidemiology, Public Health School, Harbin Medical University, Heilongjiang, China. '2Laboratory of Human Genetics, Beijing Hypertension League Institute, Beijing, China. ${ }^{3}$ The First Affiliated Hospital, Medical College of Shantou University, Shantou, China. ${ }^{4}$ Fuwai Hospital, Chinese Academy of Medical Sciences, Beijing, China.

\section{Authors' contributions}

$N Z, X L, Y W$ carried out the molecular genetic studies and drafted the manuscript. MT participated in the sequence alignment. LY, LM, SW, HZ, LL participated in the design of the study. $X L, J$ participated in the statistical analysis. XW and JZ designed the study, participated in its design and coordination, and drafted the manuscript. All authors read and approved the final manuscript.

Received: 28 September 2011 Accepted: 6 July 2012

Published: 6 July 2012

\section{References}

1. Bonita R, Mendis S, Truelsen T, Bogousslavsky J, Toole J, Yatsu F: The global stroke initiative. Lancet Neurol 2004, 3:391

2. Zhang LF, Yang J, Hong Z, Yuan GG, Zhou BF, Zhao LC, Huang YN, Chen J, Wu YF: Proportion of different subtypes of stroke in China. Stroke 2003, 34:2091-2096.

3. O'Donnell MJ, Xavier D, Liu L, Zhang H, Chin SL, Rao-Melacini P, Rangarajan S, Islam S, Pais P, McQueen MJ: Risk factors for ischaemic and intracerebral haemorrhagic stroke in 22 countries (the INTERSTROKE study): a case-control study. Lancet 2010, 376:112-123.

4. Muir KW, Tyrrell P, Sattar N, Warburton E: Inflammation and ischaemic stroke. Curr Opin Neurol 2007, 20:334.

5. Flex A, Gaetani E, Papaleo P, Straface G, Proia AS, Pecorini G, Tondi P, Pola P, Pola R: Proinflammatory genetic profiles in subjects with history of ischemic stroke. Stroke 2004, 35:2270-2275.

6. Zee RYL, Ridker PM: Polymorphism in the human C-reactive protein (CRP) gene, plasma concentrations of CRP, and the risk of future arterial thrombosis. Atherosclerosis 2002, 162:217-219.

7. Yoshida M, Takano Y, Sasaoka T, Izumi T, Kimura A: E-selectin polymorphism associated with myocardial infarction causes enhanced leukocyte-endothelial interactions under flow conditions. Arterioscler Thromb Vasc Biol 2003, 23:783-788.

8. Rubattu S, Volpe M, Kreutz R, Ganten U, Ganten D, Lindpaintner K. Chromosomal mapping of quantitative trait loci contributing to stroke in a rat model of complex human disease. Nat Genet 1996, 13:429-434.

9. Wang X, Cheng S, Brophy VH, Erlich HA, Mannhalter C, Berger K, Lalouschek W, Browner WS, Shi Y, Ringelstein EB: A meta-analysis of candidate gene polymorphisms and ischemic stroke in 6 study populations. Association of lymphotoxin-alpha in nonhypertensive patients. Stroke 2009, 108:524587-v524581. STROKEAHA.

10. Cordell HJ, Clayton DG: Genetic association studies. Lancet 2005, 366:1121-1131.
11. Benjamini $Y$, Hochberg $Y$ : Controlling the false discovery rate: a practical and powerful approach to multiple testing. Journal of the Royal Statistical Society Series B (Methodological) 1995, 289-300.

12. Smith NL, Hindorff LA, Heckbert SR, Lemaitre RN, Marciante KD, Rice K, Lumley T, Bis JC, Wiggins KL, Rosendaal FR: Association of genetic variations with nonfatal venous thrombosis in postmenopausal women JAMA 2007, 297:489.

13. Arboix A, Roig H, Rossich R, Martinez E, Garc-Eroles L: Differences between hypertensive and non-hypertensive ischemic stroke. Eur J Neurol 2004, 11:687-692.

14. Nakamura H, Luster AD, Nakamura T, In KH, Sonna LA, Deykin A, Israel E, Drazen JM, Lilly CM: Variant eotaxin: its effects on the asthma phenotype. J Allergy Clin Immunol 2001, 108:946-953.

15. Farahi N, Cowburn AS, Upton PD, Deighton J, Sobolewski A, Gherardi E, Morrell NW, Chilvers ER: Eotaxin-1/CC chemokine ligand 11: a novel eosinophil survival factor secreted by human pulmonary artery endothelial cells. J Immunol 2007, 179:1264

16. Colobran R, Pujol-Borrell R, Armengol M, Juan M: The chemokine network II. On how polymorphisms and alternative splicing increase the number of molecular species and configure intricate patterns of disease susceptibility. Clin Exp Immunol 2007, 150:1-12.

17. Haley KJ, Lilly CM, Yang JH, Feng Y, Kennedy SP, Turi TG, Thompson JF, Sukhova GH, Libby P, Lee RT: Overexpression of eotaxin and the CCR3 receptor in human atherosclerosis: using genomic technology to identify a potential novel pathway of vascular inflammation. Circulation 2000, 102:2185.

18. Kodali RB, Kim WJH, Galaria II, Miller C, Schecter AD, Lira SA, Taubman MB: CCL11 (Eotaxin) induces CCR3-dependent smooth muscle cell migration. Arterioscler Thromb Vasc Biol 2004, 24:1211-1216.

19. Cheng SS, Lukacs NW, Kunkel SL: Eotaxin/CCL11 suppresses IL-8/CXCL8 secretion from human dermal microvascular endothelial cells. J Immunol 2002, 168:2887.

20. Ortlepp JR, Vesper K, Mevissen V, Schmitz F, Janssens U, Franke A, Hanrath $P$, Weber $C$, Zerres $K$, Hoffmann R: Chemokine receptor(CCR 2) genotype is associated $\mathrm{w}$ it $\mathrm{h}$ myo-cardial infarction and heart failure in pa-tients under 65 years of age. J Mol Med 2003, 81:363-367.

21. Petrkova J, Cermakova Z, Drabek J, Lukl J, Petrek M: CC chemokine receptor (CCR)2 polymorphism in Czech patients with myo-cardial infarction. Immunol Lett 2003, 88:53-55.

22. Nyquist PA, Winkler CA, McKenzie LM, Yanek LR, Becker LC, Becker DM: Single nucleotide polymorphisms in monocyte chemoattractant protein1 and its receptor act synergistically to increase the risk of carotid atherosclerosis. Cerebrovasc Dis 2009, 28(2):124-130.

23. Dimitrijevic OB, Stamatovic SM, Keep RF, Andjelkovic AV: Absence of the chemokine receptor CCR2 protects against cerebral ischemia/reperfusion injury in mice. Stroke 2007, 38:1345-1353.

24. Mooyaart AL, Valk EJJ, van LA Es, Bruijn JA, de Heer E, Freedman BI, Dekkers OM, Baelde HJ: Genetic associations in diabetic nephropathy: a metaanalysis. Diabetologia 2011, 54:544-553.

25. Terao S, Yilmaz G, Stokes KY, Russell J, Ishikawa M, Kawase T, Granger DN: Blood cell-derived RANTES mediates cerebral microvascular dysfunction, inflammation, and tissue injury after focal ischemia Creperfusion. Stroke 2008, 39:2560-2570.

26. Mirabelli-Badenier M, Braunersreuther V, Viviani GL, Dallegri F, Quercioli A, Veneselli E, Mach F, Montecucco F: CC and CXC chemokines are pivotal mediators of cerebral injury in ischaemic stroke. Thromb Haemost 2011, 105:105.

27. Sie MPS, Uitterlinden AG, Bos MJ, Arp PP, Breteler M, Koudstaal PJ, Pols HAP, Hofman A, van Duijn CM, Witteman J: TGF-\{beta\}1 polymorphisms and risk of myocardial infarction and stroke. Stroke 2006, 37:2667-2671.

28. Iovannisci DM, Lammer EJ, Steiner L, Cheng S, Mahoney LT, Davis PH, Lauer RM, Burns TL: Association between a leukotriene C4 synthase gene promoter polymorphism and coronary artery calcium in young women. Arterioscler Thromb Vasc Biol 2007, 27:394-399.

29. Hoppe C, Klitz W, D'Harlingue K, Cheng S, Grow M, Steiner L, Noble J, Adams $R$, Styles $L$ : Confirmation of an association between the TNF $(-308)$ promoter polymorphism and stroke risk in children with sickle cell anemia. Stroke 2007, 107:483115-v483111. STROKEAHA.

30. Cui T, Wang L, Wu J, Xie J: The association analysis of FcepsilonRlbeta with allergic asthma in a Chinese population. Chin Med J (Engl), 2003:116. 
31. Nikol S, Isner JM, Pickering JG, Kearney M, Leclerc G, Weir L: Expression of transforming growth factor-1 is increased in human vascular restenosis lesions. J Clin Invest 1992, 90:1582-1592.

32. Schulick AH, Taylor AJ, Zuo W, Qiu CB, Dong G, Woodward RN, Agah R, Roberts $A B$, Virmani $R$, Dichek DA: Overexpression of transforming growth factor- $\beta 1$ in arterial endothelium causes hyperplasia, apoptosis, and cartilaginous metaplasia. Proc Natl Acad Sci U S A 1998, 95:6983-6988.

33. Donnadieu $E$, Jouvin $M H$, Kinet JP: A second amplifier function for the allergy-associated $\mathrm{Fc}(\mathbf{\epsilon}) \mathrm{Rl}-\beta$ subunit. Immunity 2000, 12:515-523.

34. Savoia C, Schiffrin EL: Inflammation in hypertension. Curr Opin Nephrol Hypertens 2006, 15:152

35. Cachofeiro V, Miana M, Martin-Fernandez B, Ballesteros S, Balfagon G, Laher $\checkmark$ : Inflammation: a link between hypertension and atherosclerosis. Current Hypertension Reviews 2009, 5:40-48.

doi:10.1186/1742-2094-9-162

Cite this article as: Zhao et al.: Association of inflammatory gene polymorphisms with ischemic stroke in a Chinese Han population. Journal of Neuroinflammation 2012 9:162.

\section{Submit your next manuscript to BioMed Central and take full advantage of:}

- Convenient online submission

- Thorough peer review

- No space constraints or color figure charges

- Immediate publication on acceptance

- Inclusion in PubMed, CAS, Scopus and Google Scholar

- Research which is freely available for redistribution 